\title{
THE ABILITY OF SMOOTH AND ROUGH STRAINS OF STREPTOCOCCUS PNEUMONIAE TO ACTIVATE HUMAN COMPLEMENT BY THE ALTERNATIVE PATHWAY
}

\author{
M. Edwards AND J. M. Stark \\ Department of Medical Microbiology, Welsh National School of Medicine \\ Cardiff CF4 $4 X N$
}

\begin{abstract}
Plate III
Although interaction with complement components facilitates the phagocytosis of pneumococci (Smith and Wood, 1969), the influence of this mechanism on the pathogenesis of lobar pneumonia is not fully defined. An understanding of this process must take into account the alternative pathway of complement activation. This pathway does not require specific antibody and, in the nonimmune host, might therefore play an important role in enhancing phagocytosis during the early phase of pneumococcal infections. Excessive activation of complement, however, may have deleterious effects through the excessive release of powerful agents, namely, the histamine-releasing anaphylatoxins and cytotoxic $C \overline{567}$ complexes. Such activation is thought to contribute to the pathogenesis of conditions such as bacteriaemia produced by Gram-negative organisms (Fearon et al., 1975), meningococcal septicaemia (Johnson and Laurell, 1975; Greenwood, Onyewotu and Whittle, 1976), quartan malaria (Greenwood and Brueton, 1974), and periodontal disease (Allison, Schorlemmer and Bitter-Suermann, 1976). Pneumococci of different serotypes have been found to vary in their ability to activate the alternative pathways in the sera of different species. This paper examines in a semi-quantitative manner the ability of three capsulate strains of Streptococcus pneumoniae, serotypes 1, 2 and 3 , and a non-capsulate strain of serotype 47 to activate human complement.
\end{abstract}

\section{MATERIALS AND METHODS}

Organisms. S. pneumoniae strains (serotypes 1,2,3 and 47) from the National Collection of Type Cultures, Central Public Health Laboratory, Colindale Avenue, London, NW9 $5 \mathrm{HT}$, were maintained by serial passage on chocolate-agar plates and were replaced at monthly intervals from reconstituted freeze-dried cultures prepared when the strains were first received. Throughout the work, the strains retained their respective smooth or rough character as judged by colonial morphology. The pneumococci were harvested from 18-h cultures grown in Bacto-Antibiotic Medium 6, Code 0666 (Difco Laboratories, P. O. Box 14B, Central Avenue, East Molesey, Surrey). Cultures (20-ml aliquots) were centrifuged at $670 \mathrm{~g}$ for $25 \mathrm{~min}$. and the cells were washed twice in isotonic saline.

Quantitation of bacterial suspensions. Washed cells were resuspended in isotonic saline and a small aliquot was taken to determine the particle concentration with a Coulter Counter, model ZB1. Suitable dilutions for this purpose were prepared in " Isoton "(Coulter

Received 1 Jan. 1977; revised version accepted 15 May 1977.

J. MED. MICROBIOL.-VOL. 11 (1977) 
Electronics Ltd, Coldharbour Lane, Harpenden, Herts, AL5 4UN), a modified Eagle's solution with $0.1 \%$ sodium azide as preservative.

To ensure that the particle count represented the same number of organisms for each serotype, the range in particle size was measured with the Coulter Counter at decreasing threshold settings. The counter threshold selected was that which gave maximal sensitivity without introducing an unacceptable number of background counts with the Isoton diluent, A setting of 8 was used (fig. 1) and was confirmed on each occasion as being the point below which the background count increased. The count therefore is a relative, reproducible expression of the total number of particles in the suspension.

The Coulter count of a culture dilution remained constant over a 1-h period. A standard suspension of $1 \times 10^{8}$ particles per $\mathrm{ml}$ was then prepared by making the appropriate dilution in isotonic saline of the sampled suspension which had been kept at $4^{\circ} \mathrm{C}$ for no longer than $30 \mathrm{~min}$. during the counting procedure.

In lightly-spread Gram-stained films of each suspension, the mean number of organisms per particle was determined by counting the number of bacteria in 40 particles in each of two films.

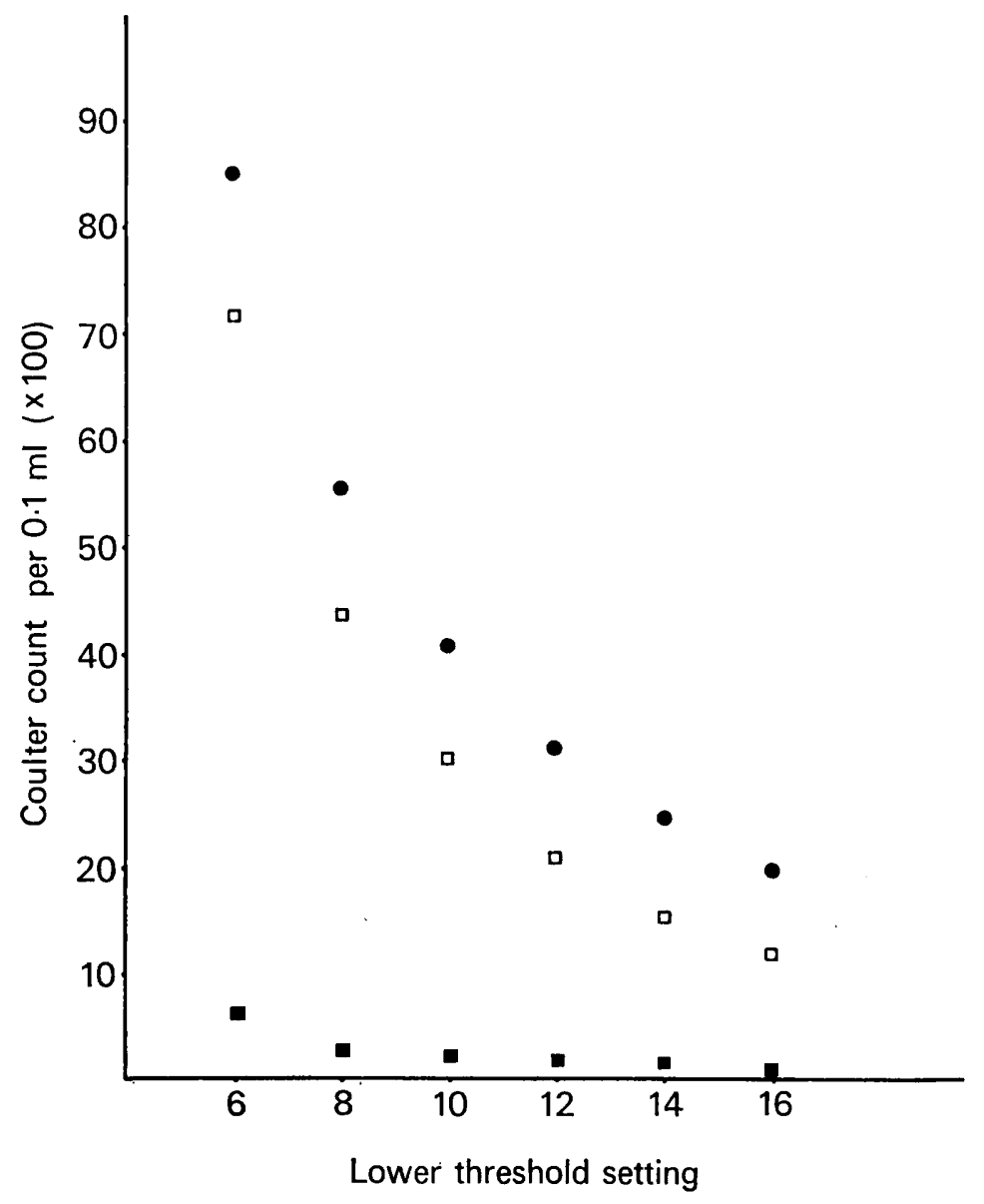

FIG. 1.-Particle sizing of Isoton suspensions of Streptococcus pneumoniae, serotypes 3 and 47. Threshold settings are arbitrary instrument units increasing with size of particle counted. Serotype $3 ; 0=$ serotype $47 ; \square=$ Isoton diluent. 


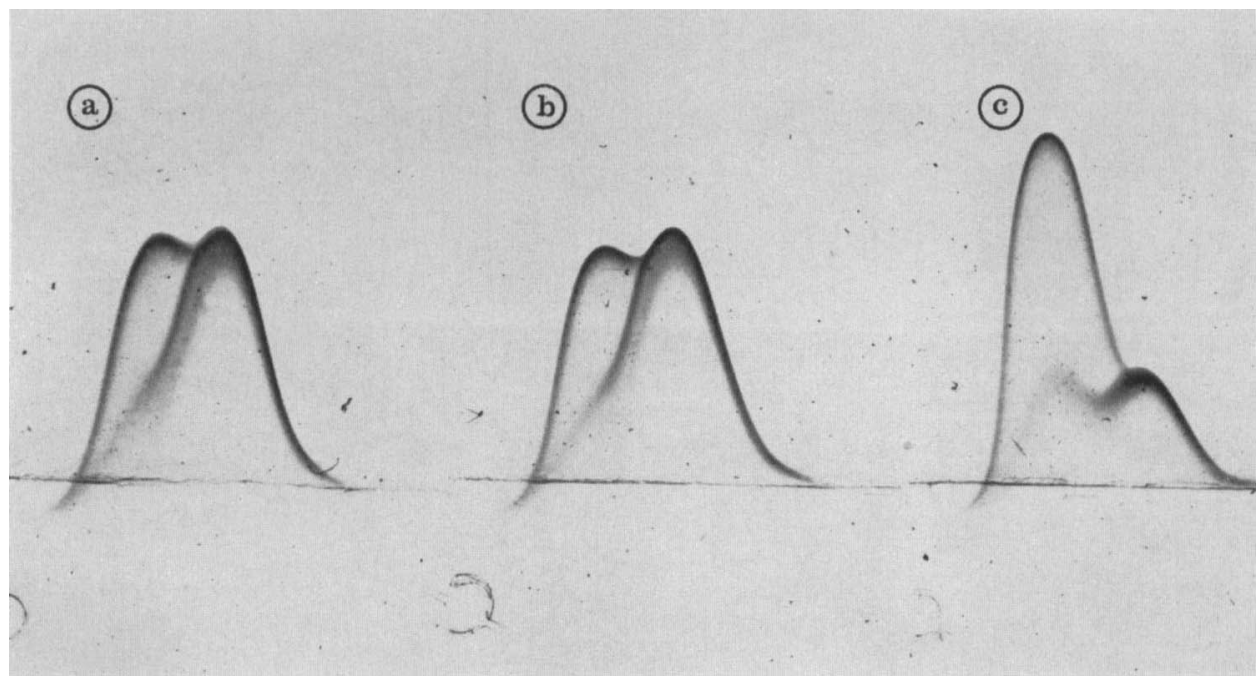

Frg. 2.-Quantitation of $\mathrm{C} 3$ activation by crossed immunoelectrophoresis. First stage of electrophoresis was from left to right. The peak on the left is the $\beta_{1 \mathrm{c}}$ form, and the peak on the right the $\beta_{1 \mathrm{~A}}$ form after activation. $a=$ Activation by serotype 3 pneumococci; $b=$ activation by serotype 47 pneumococci; $c=$ saline control. 
Human serum. Peripheral venous blood from healthy young-adult males was allowed to clot at room temperature for $30 \mathrm{~min}$. and the serum was separated by centrifugation; $1-\mathrm{ml}$ aliquots of serum were stored at $-70^{\circ} \mathrm{C}$ and thawed shortly before use. Thawed aliquots of serum were used only once.

Antiserum. Rabbit antibody (Code No. OTEA 05) to human $\beta_{1 \mathrm{c}}$ and $\beta_{1 \mathrm{~A}}$ globulin was obtained from Hoechst Pharmaceuticals, P.O. Box 18, Hounslow, Middlesex TW4 6JH.

Complement activation by pneumococcal suspensions. Pneumococci were allowed to act on complement by adding $0.04 \mathrm{ml}$ of a freshly prepared saline suspension of organisms to $0.08 \mathrm{ml}$ of serum diluted with $0.08 \mathrm{ml}$ of veronal buffered saline (Lachmann, Hobart and Aston, 1973) containing $0.025 \mathrm{M}$ magnesium chloride and $0.025 \mathrm{M}$ ethylene glycol bis(aminoethyl)-tetra acetic acid (EGTA) (Sandberg and Osler, 1971; Fine et al., 1972). This mixture was incubated at $37^{\circ} \mathrm{C}$ for $45 \mathrm{~min}$. and the conversion of complement then arrested by the addition of disodium ethylene diamino-tetra acetate (EDTA) to a final concentration of 10mm (Laurell and Lundh, 1967). The above procedure was carried out with serial dilutions of the pneumococcal suspension and a saline control was set up at the same time.

The degree of complement activation was measured by estimation of $\mathrm{C} 3$ cleavage with the crossed immunoelectrophoresis technique (fig.2), in $1.5 \%$ agarose gel containing $0.01 \mathrm{M}$ EDTA and rabbit anti-human C3 serum (Laurell, 1965 and 1966). The areas under the peaks were

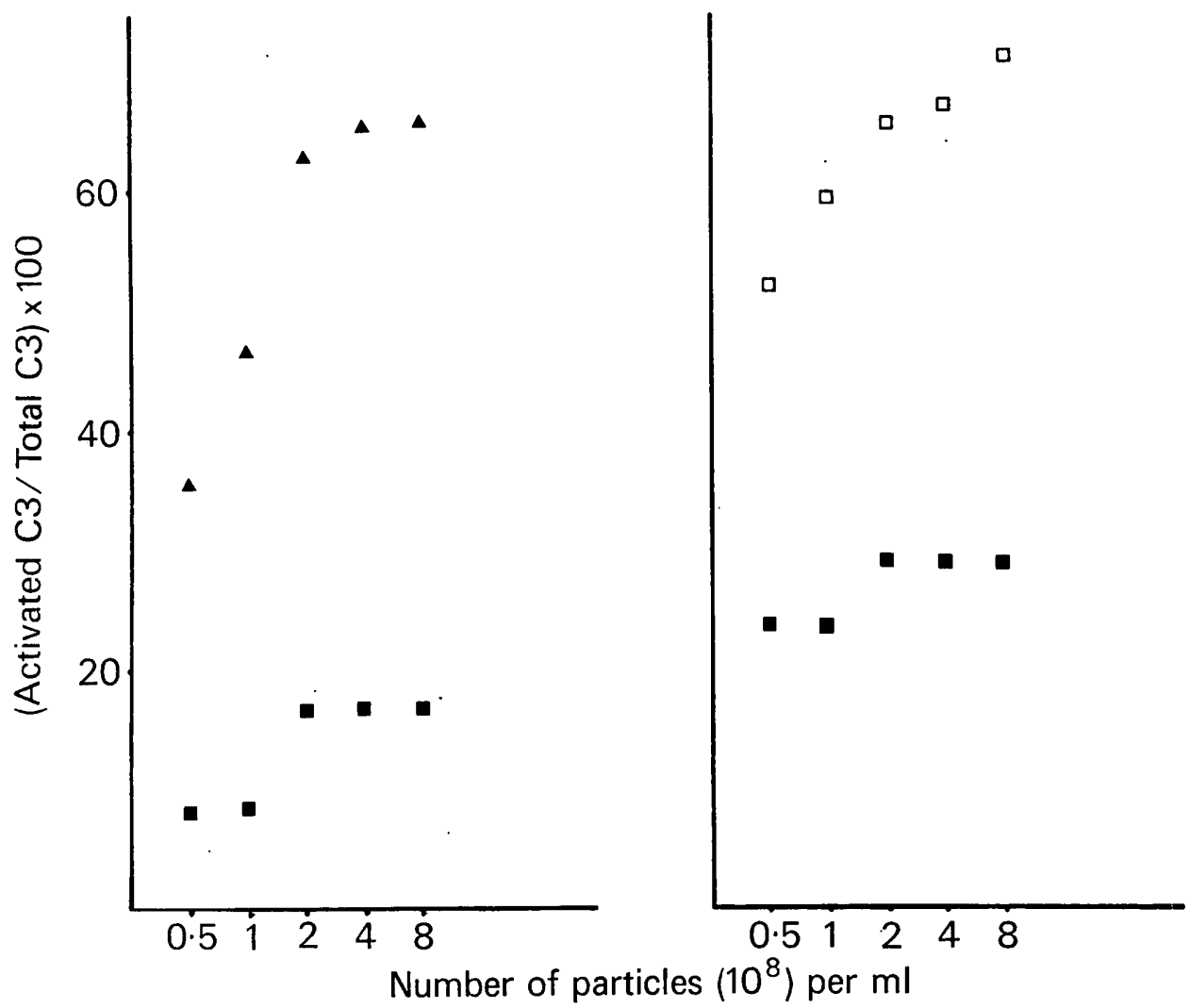

FIG. 3.-Activation of C3 by $S$. pneumoniae, serotypes 3 and 47, over a concentration range of 50-800 million particles per $\mathrm{ml}$. $\Delta=$ Serotype 3 pneumococci; $\square=$ serotype 47 pneumococci; = saline control. For each test sample a control was included in the same electrophoretic run. 
measured and the degree of cleavage expressed as the percentage of activated C3 to total C3. Because the extent of baseline $\mathrm{C} 3$ activation varies in the control samples, the activation produced by the suspension was expressed as the difference between the test and control values.

\section{RESULTS \\ Quantitation of suspensions}

Gram-stained films of a suspension of washed organisms in Isoton diluent showed that the organisms were most commonly present $(30-40 \%)$ as diplococci, the mean number of organisms per particle for serotypes $1,2,3$ and 47 being $2 \cdot 6,2 \cdot 0,2 \cdot 0$ and $2 \cdot 2$ respectively. The distribution of particle size expressed as

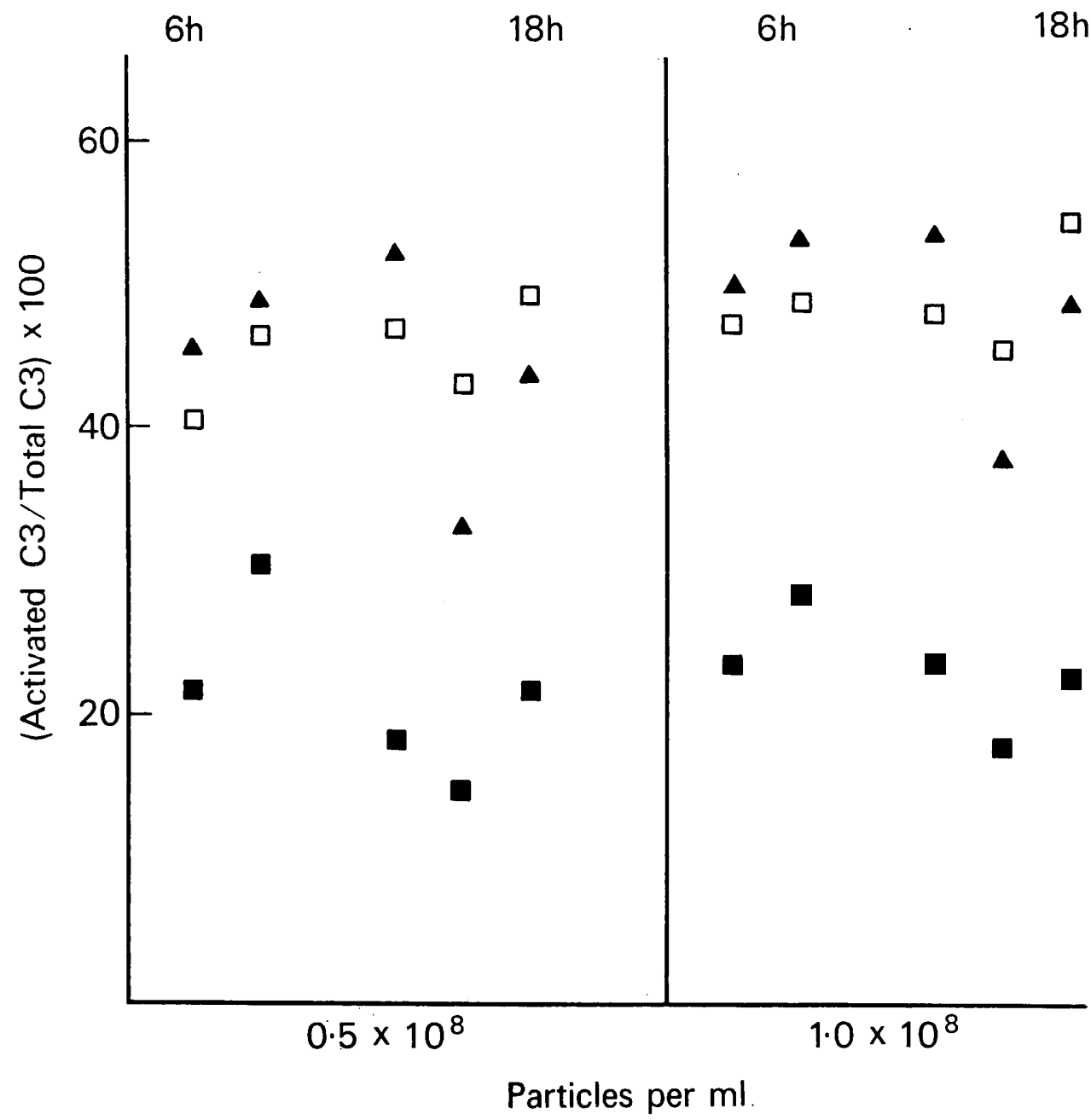

Fig. 4.-Activation of $\mathrm{C} 3$ by 6-h and 18-h (columns on left and right respectively, with each concentration of particles) cultures of $S$. pneumoniae, serotypes 3 and 47 , in concentrations of $0.5 \times 10^{8}$ and $1 \times 10^{8}$ particles per $\mathrm{ml}$. $\Delta=$ Serotype $3 ; \square=$ serotype $47 ; \square=$ saline control. For each test sample a control was included in the same electrophoretic run. 
number of organisms per cluster was similar for each serotype, over $90 \%$ of particles being represented by single or paired organisms.

A suspension of serotype 3 in Isoton had a distribution of particle size similar to that of serotype 47 as determined by the Coulter Counter (fig. 1). The particle count with each serotype was therefore taken to have the same relationship to the total number of organisms.

\section{Activation of $\mathrm{C3}$}

Preliminary experiments with serotypes 3 and 47 showed that a concentration of organisms greater than $1 \times 10^{8}$ per $\mathrm{ml}$ induced maximal activation of C3 (fig. 3). In subsequent experiments suspensions of each serotype containing (millions) $12 \cdot 5,25,50$ and 100 particles per ml did not differ significantly in their ability to activate $\mathrm{C} 3$. At a concentration of $0.5 \times 10^{8}$ per $\mathrm{ml}$ the increases in percentage activation over the saline controls were $25 \cdot 4 \pm 6.9$ and $26 \cdot 4 \pm 6 \cdot 0$ (number of samples $=5$ ) with serotypes 3 and 47 respectively. Similarly, at a concentration of $1.0 \times 10^{8}$ per $\mathrm{ml}$, the increases were $27 \cdot 4 \pm 4 \cdot 0$ and $27 \cdot 2 \pm 4 \cdot 5$ (number of samples $=5$ ) respectively (fig. 4).

Cultures harvested at $6 \mathrm{~h}$ behaved in the same manner as 18-h cultures.

The comparison of the non-capsulate strain (serotype 47) with two further smooth strains (serotypes 1 and 2) also suggested that the rough strain did not differ from these smooth strains in ability to bring about $\mathrm{C} 3$ activation (fig. 5).

\section{DISCUSSION}

Should there be a relationship between the virulence of a pneumococcal strain and its ability to activate the alternative complement pathway, the abilities of the potentially virulent capsulate serotypes 1,2 and 3 would be expected to differ from that of the avirulent rough strain, serotype 47 . Such a difference was not found in the present experiments when a range of concentrations of these organisms was tested in a human complement system deprived of $\mathrm{Ca}^{++}$ions but not $\mathrm{Mg}^{++}$ions by the chelating agent EGTA. In this system, the lack of $\mathrm{Ca}^{++}$ions prevents classical pathway activation so that any activation, measured by the conversion of $\beta_{1 \mathrm{C}}$ to $\beta_{1 \mathrm{~A}}$ can be attributed to activation by the alternative pathway rather than by the antibody-requiring classical pathway (Fine et al., 1972).

Because the virulence of the pneumococcus varies with the time of harvesting and was maximal in organisms obtained at 4-8 h in the experiments of Wood and Smith (1949), organisms harvested at both $6 \mathrm{~h}$ and $18 \mathrm{~h}$ after inoculation were examined but were not found to differ in their complement-activating ability.

Fine (1975) observing the consumption of components of human complement found that a type 1 pneumococcus was unable to activate the alternative pathway of human serum whereas types $7,12,14$ and 25 did so. Types 3,4 and 8 appeared to require specific antibody for activation. These results suggested that the ability to evade opsonisation by the alternative pathway may 


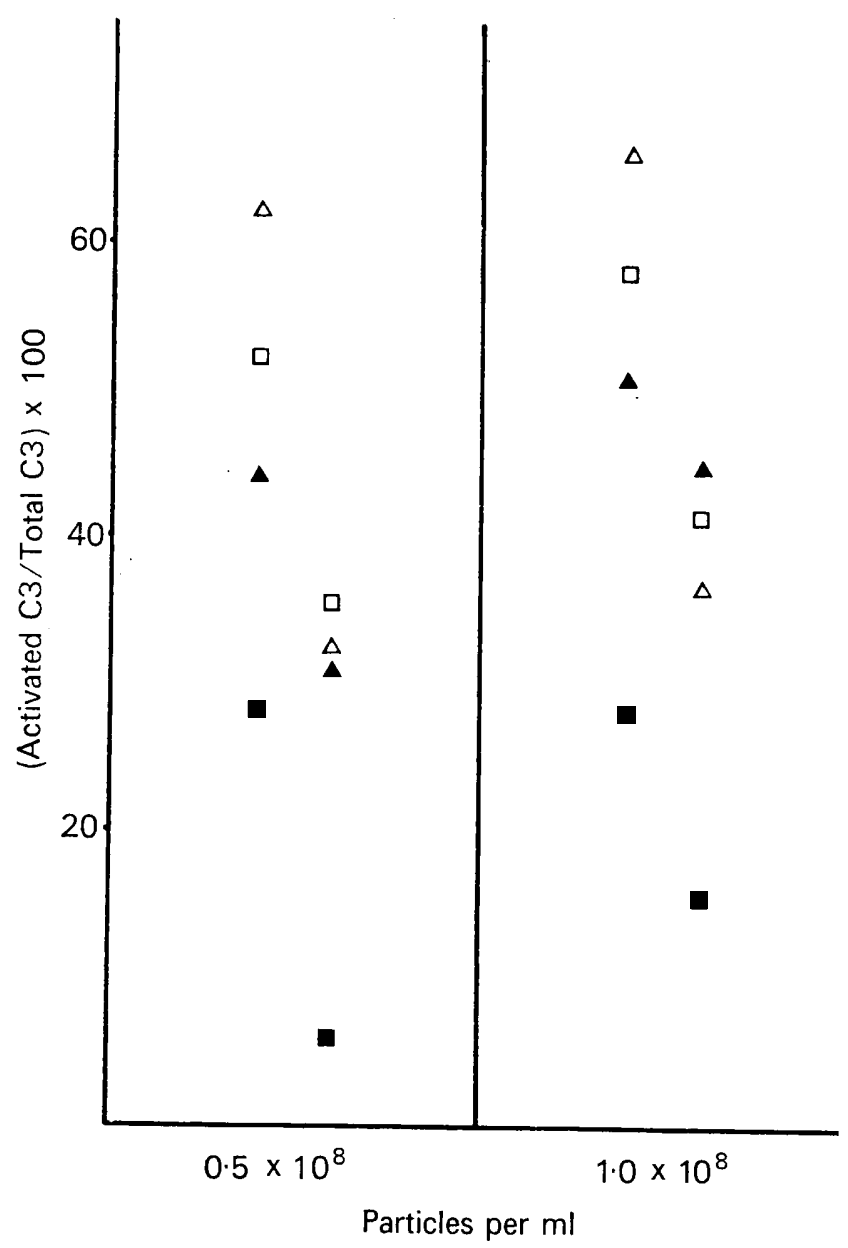

FIG. 5.-Activation of C3 by 6-h cultures of $S$. pneumoniae, serotypes 1,2 and $47 . \quad A=$ Serotype 1 pneumococci; $\square=$ serotype 2 pneumococci; $\triangle=$ serotype 47 pneumococci; $\square=$ saline control. For each test sample a control was included in the same electrophoretic run.

be related to virulence. Strain differences unrelated to capsule might account for the differences between our findings and those of Fine (1975).

Pneumococci tested in guinea-pig serum deficient in the C4 component of complement behaved similarly to those reported here (Winkelstein, Bocchini and Schiffman, 1976). A rough strain derived from a type 2 organism activated the guinea-pig alternative pathway as did the isolated polysaccharides of pneumococcal types 1,4 and 25. The polysaccharides of types 2, 3, 14 and 19, however, did not activate the alternative pathway, although the whole intact organisms from which they could be derived were able to do so. Furthermore, type 3 polysaccharide did not activate mouse-serum complement (Pryjma, Humphrey and Klaus, 1974).

A relationship has been shown between the virulence of the pneumococcus and the titre of complement-rich homologous serum with which the organism has 
to be opsonised before phagocytosis by the rat leucocyte occurs (Shin, Smith and Wood, 1969; Smith and Wood, 1969). Although in the present experiments a difference has not been demonstrated between the two types of organism, capsulate and non-capsulate, in their interaction with complement, the significance of the alternative-pathway reaction must not be overlooked. In particular important by-products of complement activation, the anaphylatoxins and $\overline{C 567}$ complexes, cause permeability changes and cell damage (Ward, Cochrane and Müller-Eberhard, 1966; Dias da Silva and Lepow, 1967; Cochrane and MüllerEberhard, 1968) and these might be involved in pathogenicity. There is indeed in-vivo evidence to suggest that the alternative pathway may be activated during the early phase of a pneumococcal pneumonia (Reed, Davidson and Williams, 1976).

Because the virulent organism is less readily phagocytosed and requires a greater degree of complement binding for opsonisation, its relative persistence in contrast to that of the avirulent organism would be expected to cause excessive release of acute inflammatory mediators. As the pneumococcus does not synthesise a potent toxin, these mediators might be responsible for the astonishing wave of oedema so characteristic of pneumococcal lobar pneumonia.

\section{SUMMARY}

Three capsulate pneumococcal strains of serotypes 1,2 and 3, and one noncapsulate strain of serotype 47 , were found to activate human complement by the alternative pathway to a similar extent over the concentration range examined. Nevertheless, the capsulate strains, in contrast to the non-capsulate, are known to require complement attachment for phagocytosis and it is therefore postulated that the toxic by-products released cause the wave of oedema characteristic of pneumococcal lobar pneumonia.

\section{REFERENCES}

Allison, A. C., Schorlemmer, H. U. and Bitter-Suermann, D. 1976. Activation of complement by the alternative pathway as a factor in the pathogenesis of periodontal disease. Lancet, 2, 1001.

Cochrane, C. G. and Müller-Eberhard, H. J. 1968. The derivation of two distinct anaphylatoxin activities from the third and fifth components of human complement. J. exp. Med., 127, 371.

Dias da Silva, W. ANd Lepow, I. H. 1967. Complement as a mediator of inflammation. II. Biological properties of anaphylatoxin prepared with purified components of human complement. J. exp. Med., 125, 921.

Fearon, D. T., Ruddy, S., Schur, P. H. and McCabe, W. R. 1975. Activation of the properdin pathway of complement in patients with Gram-negative bacteremia. N. Engl. J. Med., 292, 937.

FINE, D. P. 1975. Pneumococcal type-associated variability in alternate complement pathway activation. Infect. Immun., 12, 772.

Fine, D. P., Marney, S. R. JR, Colley, D. G., Sergent, J. S. and Des Prez, R. M. 1972. C3 shunt activation in human serum chelated with EGTA. J. Immun., 109, 807.

Greenwood, B. M. ANd Brueton, M. J. 1974. Complement activation in children with acute malaria. Clin. exp. Immun., 18, 267. 
Greenwood, B. M., Onyewotu, I. I. ANd Whittle, H. C. 1976. Complement and meningococcal infection. Br. med. J., 1, 797.

Johnson, U. AND LAUReLL, A. 1975. Complement activation in meningococcal septicaemia. Acta path. microbiol. scand., C 83, 285.

Lachmann, P. J., Hobart, M. J. And Aston, W. P. 1973. Complement technology. In Handbook of experimental immunology, 2nd ed., edited by D. M. Weir Oxford, London, Edinburgh and Melbourne, vol. 1, chapter 5.

LAURELl, C. B. AND LUNDH, B. 1967. Electrophoretic studies of the conversion products of serum $\beta_{1 \mathrm{c}}$-globulin. Immunology, 12, 313.

LAURell, C. B. 1965. Antigen-antibody crossed electrophoresis. Analyt. Biochem., 10, 358.

LAURELL, C. B. 1966. Quantitative estimation of proteins by electrophoresis in agarose gel containing antibodies. Analyt. Biochem., 15, 45.

Pryjma, J., Humphrey, J. H. AND Klaus, G. G. B. 1974. C3 activation and independent B cell stimulation. Nature, Lond., 252, 505.

Reed, W. P., Davidson, M. S. and Williams, R. C. 1976. Complement system in pneumococcal infections. Infect. Immun., 13, 1120.

SANDBerg, A. L. AND Osler, A. G. 1971. Dual pathways of complement interaction with guinea pig immunoglobulins. J. Immun., 107, 1268.

Shin, H. S., SMITH. M. R. AND Wood, W. B. JR 1969. Heat labile opsonins to pneumococcus. II. Involvement of C3 and C5. J. exp. Med., 130, 1229.

Smith, M. R. AND Wood, W. B. JR 1969. Heat labile opsonins to pneumococcus. I. Participation of complement. J. exp. Med., 130, 1209.

Ward, P. A., Cochrane, C. G. ANd Müller-Eberhard, H. J. 1966. Further studies on the chemotactic factor of complement and its formation in vivo. Immunology, 11, 141.

Winkelstein, J. A., Bocchini, J. A. JR AND Schiffman, G. 1976. The role of the capsular polysaccharide in the activation of the alternative pathway by the pneumococcus. J. Immun., 116, 367.

Wood, W. B. JR AND SMIth, M. R. 1949. The inhibition of surface phagocytosis by the capsular "slime layer" of pneumococcus Type III. J. exp. Med., 90, 85. 